\title{
LIDERAZGO, MOTIVACIÓN Y APRENDIZAJE EN MICROEMPRESARIOS DE LA REGIÓN DEL MAULE, CHILE
}

\section{LEADERSHIP, MOTIVATION AND LEARNING IN SMALL BUSINESS ADMINISTRATION OF THE REGION OF THE MAULE, CHILE}

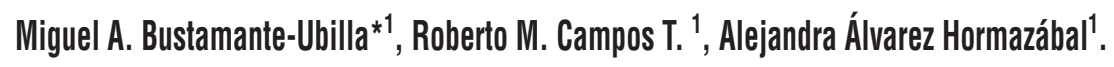

1 Facultad de Economía y Negocos, Universidad de Talca, Dos Norte 685, 3465548 Talca, Chile. E-mail: mabu@utalca.cl, rcampos@utalca.cl, ale.alvarez.hor@gmail.com

RESUMEN

ABSTRACT

En este trabajo se caracterizan los modelos mentales que presentan los microempresarios en términos de liderazgo, motivación y aprendizaje. Se aplicaron dos cuestionarios para caracterizar los estilos de liderazgo, un tercer cuestionario para determinar los estilos de aprendizaje y un cuarto instrumento para determinar los motivos de logro, poder y afiliación. Todos medidos en escala Likert. Se realizó un análisis descriptivo de frecuencias absolutas y relativas además de un análisis bivariado e inferencial de correlaciones y prueba ji-cuadrado. Complementariamente se utilizó correlación de Spearman para determinar asociación entre las variables. Se determinó que la mayor parte de los entrevistados tiende hacia un estilo de liderazgo participativo, se ubica en la categoría de experimentación activa muestra un estilo divergente. Sin embargo, aunque existen similitudes de estilos de liderazgo entre los microempresarios, no presentan la misma importancia. Las prácticas de aprendizaje más frecuente son, además del divergente el acomodador y, la mayor motivación se relaciona con el logro.

Palabras clave: Liderazgo, aprendizaje, motivación, microemprendedores.

In this work the mental models are characterized that present the microempresarios in terms of leadership, motivation and learning. Two questionnaires were applied to characterize the styles of leadership, a third questionnaire to determine the learning styles and a fourth instrument to determine the achievement reasons, to be able to and affiliation. All measured in scale Likert. She/he was carried out a descriptive analysis of absolute and relative frequencies besides an analysis bivariado and inferencial of correlations and test ji-square. Complementarily correlation of Spearman was used to determine association among the variables. It was determined that most of the interviewees spreads toward a style of leadership participativo, it is located in the category of experimentation active sample a divergent style. However, although similarities of styles of leadership exist among the microempresarios, they don't present the same importance. The practices of more frequent learning are, besides the divergent one the usher and, the biggest motivation is related with the achievement.

Keywords: Leadership, learning, motivation, small companies. 


\section{INTRODUCCIÓN}

El psicólogo escocés Kenneth Craik (1940) define los modelos mentales como "imágenes, supuestos e historias que tenemos en la mente acerca del mundo, de nosotros mismos, de los demás, de las instituciones y de todos los aspectos del mundo, los cuales influyen en nuestro comportamiento y en el proceso de decisiones y sin ellos no podríamos enfrentarnos al entorno”. Así mismo, Peter Senge (1990) afirma que son "supuestos hondamente arraigados, generalizaciones e imágenes que influyen sobre nuestro modo de comprender el mundo y actuar”.

Por otra parte, Stephen P. Robbins (1993), define el liderazgo como "... la capacidad para influir en un grupo con objeto de que alcancen las metas", lo cual no implica que los modelos mentales del líder ayudan a la toma de decisiones y a la eficacia organizacional (Senge, 1990).

Las características más comunes de los líderes son la inteligencia, habilidad de supervisión, iniciativa, seguridad en si mismo y auto-percepción del nivel ocupacional (Avolio y Bass, 2004), que se expresa en estilos rigurosos, autoritarios y estructurados (Barbosa, et al., 2017) que ubican al líder en un punto intermedio entre el líder riguroso y cercano a sus subalternos (Cabaleiro, et al., 2017). Surge así el liderazgo continuo, que depende del líder, de los seguidores y de la situación sobre la que se actúa (Duran y Castañeda, 2015).

La situación da pie a los modelos de contingencia (Fred Fiedler, 1987) que miden la escala de "el compañero de trabajo menos deseado" (Garcia, 2015) e incluso al colaborador menos deseado, llegando a establecer ocho posiciones de grupo, donde una situación muy favorable o muy poco favorable, requiere de un líder orientado al trabajo; pero si la situación tiende a ser moderadamente favorable, entonces es más conveniente contar con un líder orientado a las relaciones (Gil e Ibarra, 2014 a; 2014 b).

La situación genera a su vez el liderazgo situacional (Wegner, 2004), de contingencia, que se define a partir de la madurez relativa de los seguidores y su nivel de preparación.
Son los seguidores quienes aceptan o rechazan al líder y donde, independientemente del comportamiento del líder, la eficacia depende de las acciones de los seguidores (Zárate y Matviuk, 2012).

La escuela clásica de la organización científica del trabajo buscaba incrementar la productividad y dónde a cada función esencial correspondía una cierta capacidad específica del hombre (Dvir, et al., 2002). La Escuela Neoclásica, pone el énfasis en la participación y compromiso de los implicados puesto que el ser humano no sólo acepta responsabilidad, sino que también la busca (Ehrhart, 2004) y es capaz de modelar su comportamiento hacia sus subordinados (Escalona-Barbosa y Hurtado-Ayala, 2016).

Se precisa entonces analizar el aprendizaje (David Kolb, 1977) en relación con los modelos mentales (Senge, 1990), sobre la base de capacidades tales como la experiencia concreta (EC), observación reflexiva (OR), conceptualización abstracta (CA) y experiencia activa (EA), que determinan los estilos divergente, asimilador, acomodador y convergente. Sin embargo, el aprendizaje no es lineal, puede ser estimulado (Perez, 2012) por activadores que generan un efecto operante (Pérez y Camps, 2011), mediante reforzamiento de la conducta, que depende, a su vez, de interrelaciones y reciprocidades impulsadas por fuerzas internas, disposición a estímulos, procesos vicarios, simbólicos y auto-regulatorios del comportamiento (Bandura, 1982).

Complementariamente, las prácticas innovadoras del liderazgo (Espinosa, et al., 2015), ayudan al colectivo a activar sus competencias individuales y colectivas (Godoy, R, y Bresó, 2013) contribuyendo al logro de las metas de la organización (Li y Liao, 2014).

Sobre la base de la literatura analizada, el presente trabajo caracteriza los modelos mentales a partir del liderazgo, motivación y capacidades de aprendizaje de los microempresarios pertenecientes a una incubadora de negocios de Chile.

\section{METODOLOGÍA}

Se utilizó el método de encuesta sobre la base de cuatro cuestionarios previamente 
validados, el primero caracteriza los estilos de liderazgo (Chester Barnard, 1938), y contiene 10 pares de afirmaciones que se responden mediante una escala de o a 10 a cada par. El segundo determina los estilos de Liderazgo (Hersey \& Blanchard, 1982) y cuenta con cuatro grupos de afirmaciones, donde la alternativa elegida asigna puntaje a un estilo determinado.

El tercero caracteriza los estilos de aprendizaje (David Kolb, 1977) contiene nueve grupos de palabras que caracterizan cuatro métodos definidos por series de cuatro palabras, que se califican asignando el número 1 a la palabra que menos identifica el estilo y 4 a la que más identifica el estilo del entrevistado.

Finalmente, el cuarto, determina el estado de motivación (Robbins, 1993), en términos de logros, poder y afiliación, contiene 15 afirmaciones que el encuestado califica en escala Likert de 5 puntos que van desde totalmente de acuerdo a totalmente en desacuerdo, produciendo una columna de agregación cuya suma se ubica entre 5 y 25 puntos para determinar la necesidad dominante. La confiabilidad de los instrumentos se calculó mediante la aplicación del alfa-cronbach (a) (Eyssautier, 2006).

Se realizó un censo (Hernández, et al., 2006) de los microempresarios inscritos en un programa "Incubadora de Microempresas de Chile”, provenientes de las áreas vitivinícola, hortofrutícola, forestal y de servicios de la Región del Maule. Para la recolección de datos se acudió a las cuatro primeras sesiones de trabajo colectivo del proyecto Incubadora de Micro-Empresas. Algunas, con más de un dueño, se optó por recoger las percepciones de todos (Eyssautier, 2006).

Se realizó un análisis descriptivo de frecuencias absolutas y relativas a través del programa Excel y análisis bivariado e inferencial de correlaciones y prueba ji-cuadrado, mediante el programa SPSS 2OV (IBM, 2013).

\section{ANÁLISIS DE COVARIANZA}

Para el análisis se utilizó la correlación de Spearman (rs) que permite medir las relaciones de dos o más variables, proporciona la magnitud de asociación entre las varia- bles mediante magnitudes que van entre los números -1 y 1 (Hernández, et al. 2006). Por consiguiente, cuanto mayor sea el valor absoluto de la correlación, mayor será la asociación lineal entre las variables estudiadas.

Tabulando los datos de acuerdo a la codificación de las respuestas en una planilla Excel y los resultados obtenidos se ingresaron al programa SPSS. Se elaboró una matriz con cada una de las variables en estudio, incluyendo todas las respuestas de los cuestionarios. Se aplicó el análisis de correlación de Spearman a cada una de las respuestas para lograr la asociación entre ellas (Eyssautier, 2006. Se seleccionó la matriz las correlaciones más significativas, de acuerdo con el criterio de seleccionar las correlaciones más altas y significativas en cada análisis. Finalmente, se construyeron "árboles de correlaciones", para obtener un análisis gráfico de las relaciones determinadas.

\section{CONTEXTUALIZACIÓN}

El trabajo se desarrolló en el contexto micro - empresarial de una incubadora de negocios donde se comparten instalaciones físicas, servicios administrativos y orientación empresarial.

El programa, les otorgó un período de acompañamiento y de asentamiento de mediano plazo, ubicándolos en un espacio protegido que les proporcionaba facilidades de capacitación para la consolidación y creación de innovaciones empresariales en sus respectivos giros de negocio. La incubadora se definió como un centro difusor de tecnología, de entrenamiento empresarial y de intercambio de información tecnológica para el desarrollo y crecimiento empresarial.

\section{RESULTADOS}

A continuación, se sintetizan los hallazgos de investigación para cada una de las dimensiones de liderazgo, aprendizaje y motivación de los microempresarios de la región del Maule, de Chile.

\section{ESTILOS DE LIDERAZGO}

Se observa en la figura 1, que casi la totalidad de los microempresarios de la incubadora se encuentran motivados hacia el trabajo, mar- 
cando una tendencia definida hacia la teoría Y de McGregor, con un 94\% de microempresarios que ven el trabajo tan natural como el descanso o el juego, por el contrario, un escaso 6\% de ellos se sienten representados por la teoría X que consideran que el trabajo es desagradable $y$, en consecuencia, tratan de evitar responsabilidades.

El estilo de liderazgo, de acuerdo con la madurez de los seguidores y su nivel de preparación muestra que el $62 \%$ de los encuestados debería tender a un estilo de liderazgo "participativo" (baja tarea y alta relación), es decir, el líder y su seguidor de manera conjunta toman decisiones y la función primordial del líder es comunicar y facilitar las cosas.

Respecto a la madurez de los seguidores ( $\left.\mathrm{R}_{3}\right)$ se observan problemas motivacionales, dónde las personas son capaces pero no están dispuestos a realizar lo que quiere el líder (pueden pero no quieren), en consecuencia se hace necesario un estilo apoyador y participativo.

Se observa además, que un $26 \%$ requiere de un estilo de liderazgo ubicado en el rango "delegar" (baja tarea y baja relación), según el cual el líder proporciona poca dirección y apoyo, es decir, entrega toda la responsabilidad hacia sus subordinados debido a que su madurez es alta. Por otra parte $\left(\mathrm{R}_{4}\right)$ demarca una conducta de personas capacitadas y dispuestas a realizar lo que se les pide y tienen la capacidad de asumir responsabilidades (pueden y quieren).

Se identifica así mismo un 9\% de microempresarios que debería tener a un tipo de liderazgo clasificado en la técnica de "vender" (alta tarea y alta relación), es decir, el líder se comporta como director y entrega apoyo, las personas demuestran tener una madurez (R2), donde las personas no cuentan con las habilidades apropiadas, pero están motivadas a realizar tareas necesarias para el puesto (quieren pero no pueden).

Por último un 3\% de los entrevistados debería poseer el estilo de liderazgo clasificado en la posición de "ordenar" (alta tarea y baja relación), por lo tanto, el líder define los roles a sus subordinados, ya que estos presentan una madurez baja, R1, pues ellos necesitan instrucciones claras y específicas porque no tienen la confianza en sí mismos y por lo tanto no están dispuestos a asumir la responsabilidad de hacer algo (no quieren y no pueden) (Figura 1)

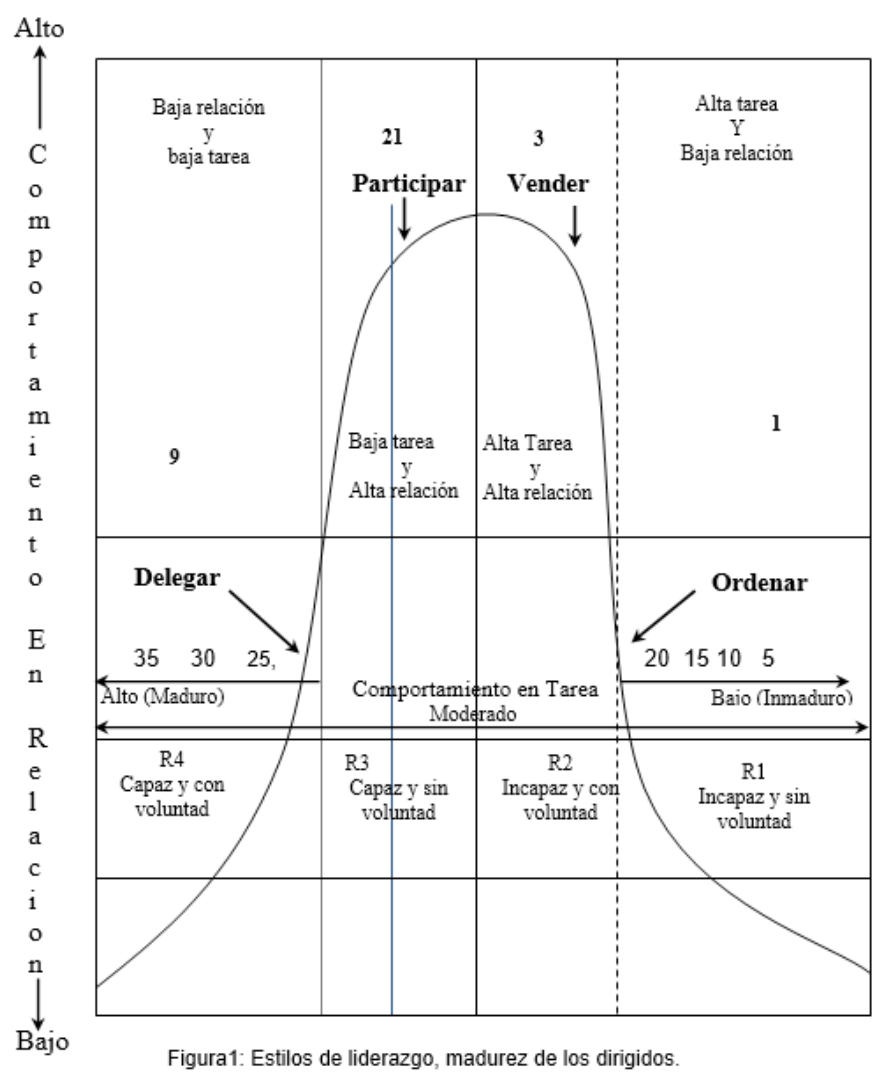




\section{MOTIVACIÓN}

Se observa en la figura 1, que un $73 \%$ de los microempresarios se sienten motivados por los logros que se definen como el impulso de sobresalir, de tener logros en relación con un conjunto de normas y de luchar por tener éxito.

La necesidad de afiliación es representada por un 9\% que se define como el tener relaciones interpersonales amistosas y cercanas, además, un $6 \%$ de los microempresarios se siente motivado por la necesidad de poder, el cual se refiere a la necesidad de hacer que otros se comporten de determinada manera, diferente a como hubieran actuado, es decir, la necesidad de influir en el comportamiento de otros.

Por otra parte, se observan algunos casos de microempresarios que se sienten motivados por dos de las variables e incluso por las tres, entre estas se cuentan la relación de logro y afiliación representada por un 3\%, lo que indica que estas personas luchan por tener éxito, cumplir metas y además por establecer relaciones interpersonales con el resto de sus compañeros de trabajo; un 3\% de la frecuencia agrupa a las personas motivadas tanto por el poder como la afiliación, y finalmente un $6 \%$ nos indica a los microempresarios que están motivados por las tres variables en conjunto.

\section{CAPACIDADES DE APRENDIZAJE}

Se observa en la figura 2, que la mayor tendencia se observa en la categoría de aprendizaje de Experimentación Activa con un $43 \%$, lo cual refleja que la mayoría de los microempresarios aprenden haciendo cosas; un $18 \%$ corresponde a la tendencia de Experiencia Concreta, que es aprender basado en la experiencia y juicios intuitivos; un $12 \%$ de la tendencia de Observación Reflexiva, donde los microempresarios aprenden probando en forma parcial y reflexiva.

Por otra parte, dos proporciones idénticas de los microempresarios (9\%) muestran tendencias combinadas. Un grupo combina las tendencias Experimentación Activa y Experiencia Concreta y un segundo grupo combina las tendencias de Conceptualización Abstracta y Experimentación Activa: En ambas los microempresarios asocian lo reflexivo con lo abstracto.

Complementariamente, un 6\% refleja la tendencia de Conceptualización Abstracta, donde el microempresario tiende al análisis y a la conceptualización, a aprender basado en el pensamiento lógico y a la evaluación racional y, un 3\% combina las tendencias Experiencia Concreta y Observación Reflexiva, donde el microempresario aprende de la experiencia y en forma reflexiva.

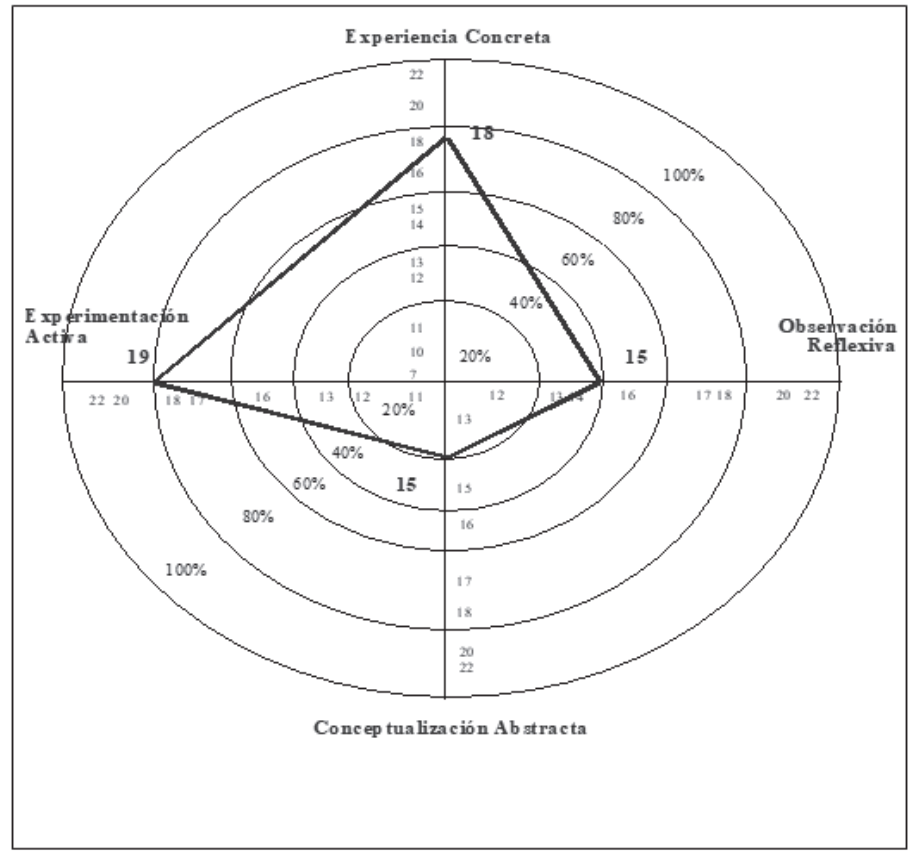

Figura 2: Estilos de aprendizaje 


\section{ESTILOS DE APRENDIZAJE}

Se determinó que un 38\% de los microempresarios entrevistados muestra un estilo divergente, según el cual las personas tienen una alta capacidad imaginativa, tienen muchas ideas, son muy sensibles y se preocupan por las personas, su debilidad es que el tener muchas ideas les dificulta tomar decisiones.

Un $29 \%$ posee un estilo de aprendizaje acomodador, esto implica que los microempresarios tienen como punto fuerte la solución de problemas, toman decisiones y riesgos a la vez, son líderes, su debilidad es que muchas veces resuelven problemas sin importancia.

Un $12 \%$ posee un estilo de aprendizaje asimilador, el cual tiene como punto fuerte la creación de modelos teóricos, se interesa menos por las personas y por la aplicación práctica de las teorías, una de sus debilidades es que tiende a construir castillos en el aire porque no sabe aplicar teorías en la práctica. Prefiere trabajar solo porque piensa que el resto de las personas le estorban.

Un $12 \%$ corresponde a una relación entre todos los estilos de aprendizaje, por lo tanto, el microempresario adapta su estilo de acuerdo al entorno en el que se encuentre (como un “camaleón”).

Por último el estilo convergente con un $9 \%$, es el más eficaz, en tanto representa a personas que prefieren tratar con cosas más que con personas y tienden a ser un tanto insensibles. Su punto fuerte es la aplicación práctica de las ideas. Las personas que poseen este estilo son las que solucionan los problemas. Su debilidad es que al ser muy apresurados pueden llegar a solucionan mal los problemas, pero rápidamente buscan otra solución y la llevan a cabo.

\section{ANÁLISIS DE CORRELACIONES}

A continuación se presentan los resultados y árboles de correlación del liderazgo, aprendizaje y motivación.

\section{CORRELACIONES DE LA VARIABLE LIDERAZGO}

Se observa en estilos de liderazgo para con los subordinados, que las afirmaciones pertenecientes a motivación y responsabilidad están relacionadas en un $48,6 \%$, lo que nos indica que ambas se inciden mutuamente en dicha magnitud. Se interpreta así mismo que el líder cuenta con la opción de ordenar, persuadir, delegar o participar con sus trabajadores, para motivar a sus colaboradores a partir de la responsabilidad observada en ellos. Se observa además, que la mayoría se inclina hacia un liderazgo participativo compartiendo la toma de decisiones y preocupándose por el problema motivacional que presentan los subordinados.

Por otra parte al relacionar los estilos de liderazgo, la teoría X, según la cual el individuo ve el trabajo como algo negativo, respecto de la teoría $\mathrm{Y}$, que indica que el individuo ve el trabajo como algo positivo, los resultados en términos de correlación es alta pero inversa (-o.813), indica que los estilos son claramente opuestos, de modo que un incremento en $\mathrm{Y}$, reduce el impacto de $\mathrm{X}$. Esto indica entonces, que los microempresarios se identifican más bien con la teoría Y, es decir la opción más positiva del trabajo y, por otra, no concuerdan o aprecian, en igual grado, los principios de la teoría X al momento de ejercer su liderazgo. En consecuencia, los microempresarios perciben que sus empleados ven positivamente el trabajo y es por eso mismo que, casi la totalidad de ellos presenta un estilo de liderazgo participativo, según el cual poseen una alta relación con sus subordinados, dejando que éstos, en sus respectivos rangos de autonomía, puedan participar en la toma de decisiones.

Respecto de liderazgo para con los subordinados, la afirmación, positiva, "Si los empleados tienen acceso a las informaciones que necesitan, suelen tener mejores actitudes y comportarse con más responsabilidad" se relaciona en grado relevante con pero de manera inversa $(-0.838)$ con la expresión, negativa "Si los empleados tienen más información de la que necesitan para sus tareas inmediatas, suelen em- 
plearlas mal”. Esta relación inversa entre un reactivo promisorio como el primero, reduce el efecto adverso del segundo reactivo, lo cual significa que, a mayor acceso de parte de los trabajadores a la información que necesitan, se estarían informan- do adecuadamente y en consecuencia, no tendrían más información que la necesaria para ejecutar sus desempeño $y$, por lo tanto, no la emplearían de mala manera o en forma inadecuada.

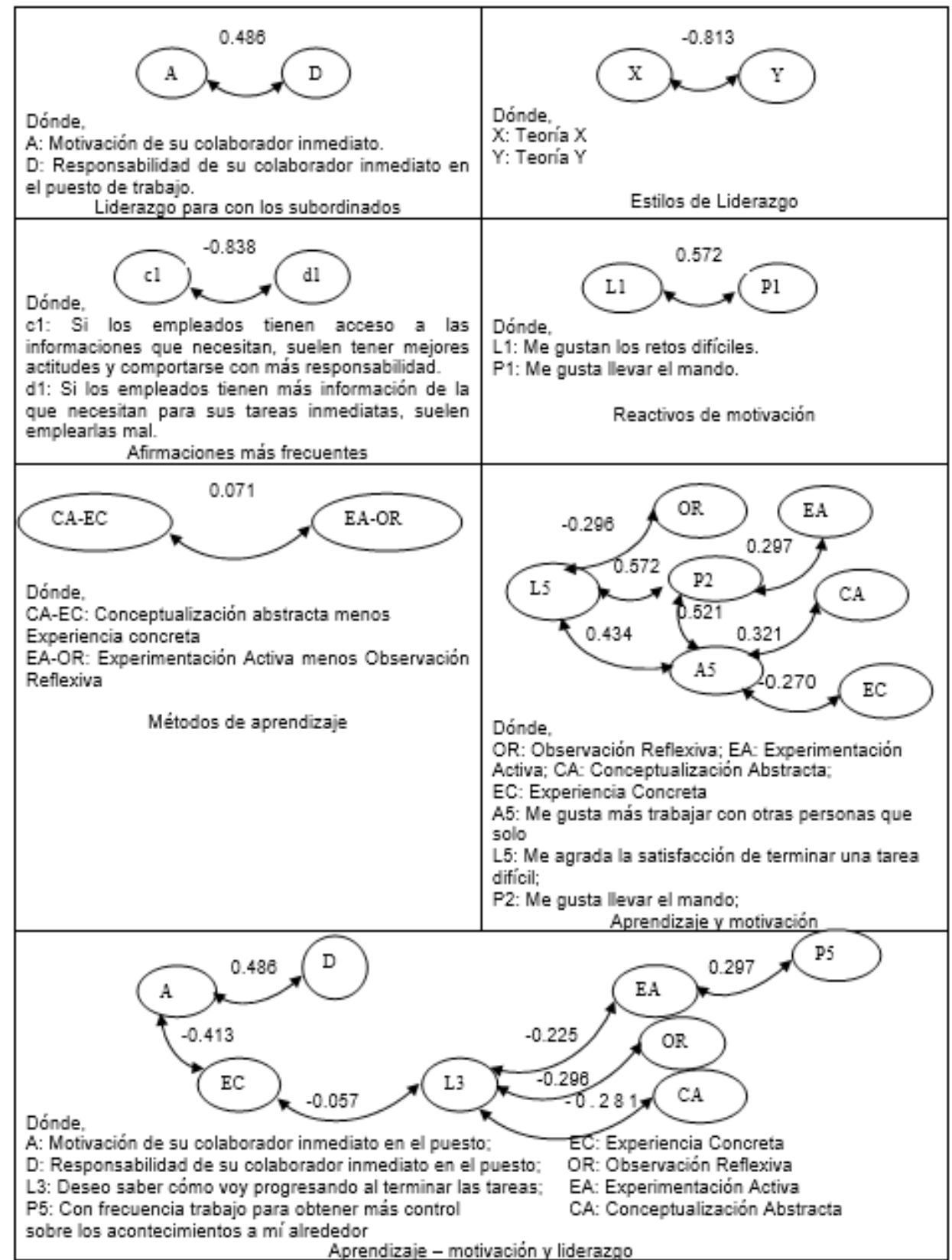

Figura 3: Árbol de correlaciones entre liderazgo y aprendizaje

Se observa en el cuadrante de reactivos de motivación, que la afirmación "Me gustan los retos difíciles", que corresponde a la motivación por los "logros", está directamente correlacionada $(0,572)$ con la afirmación "Me gusta llevar el mando", afirmación identificada con la motivación por el "po- der”. Lo que permite concluir que existen microempresarios que logran sus metas, definitivamente a través del poder.

En relación con el aprendizaje, se observan diferencias claras entre los métodos de aprendizaje, que se muestra en la rela- 
ción "Conceptualización abstracta menos Experiencia concreta" respecto de "Experimentación Activa menos Observación Reflexiva", porque poseen una muy baja correlación $(\mathrm{o}, 071)$. Este índice es sin embargo, muy favorable para explicar y concluir cuál es el estilo de aprendizaje que caracteriza a los microempresarios. Porque cada estilo de cuenta de un vector de comportamiento independiente o diferenciado, que permite apreciar claramente los estilos.

En esta relación de estilos, se determina que la mayor parte de los microempresarios se identifica con un estilo de aprendizaje y, la parte opuesta, se identificara con el otro. En consecuencia, el índice define lo relevante que es cada uno de los estilos de aprendizaje para cada grupo de microempresarios, puesto que los caracteriza con claridad como segmentos separados e independientes. Es por ello que éste puede ser considerado un hallazgos determinante puesto que los microempresarios presentan conductas extremas de forma tal que cada persona se identifica con claridad más con un método para abordar su aprendizaje que con otro, lo cual da origen a un determinado estilo característico de la persona, debido a que cada quien se ubica en un punto de intersección perfectamente determinado.

Podemos observar que existe una relación inversa de la motivación generada por los logros de los microempresarios que se expresa en "Me agrada la satisfacción de terminar una tarea difícil" respecto del método de aprendizaje "observación reflexiva", lo que nos dice que los microempresarios consiguen sus logros no por medio de la observación sino que, lo hacen de manera activa, es decir, probando en forma empírica.

El comportamiento descrito, se ratifica luego en la correlación que muestra la expresión "Me gusta llevar el mando" que incide en forma directa (0.297) sobre "Experimentación Activa” y en la relación del reactivo "Me gusta más trabajar con otras personas que solo" que incide directamente $(0,321)$ sobre "Conceptualización Abstracta" e inversamente $(-0,270)$ sobre
"Experiencia Concreta"; eventualmente como expresión del poder, valorado como un componente directivo que presenta una baja relación con la experimentación activa, donde los microempresarios aprenden haciendo cosas. Sin embargo, la correlación más fuerte entre aprendizaje y motivación del microempresario se observa entre afiliación y conceptualización abstracta, lo que indicaría que los microempresarios tienden al análisis por medio de las relaciones interpersonales amistosas y cercanas.

Por último, se observa en el cuadrante de análisis general de Aprendizaje - motivación y liderazgo, que la afirmación "Deseo saber cómo voy progresando al terminar las tareas", que identifica la motivación por los logros, muestra claramente una relación inversa respecto a los métodos de aprendizaje Experimentación activa, Experiencia concreta, Observación reflexiva y Conceptualización abstracta, lo que indica que los métodos de aprendizaje se comportan de manera opuesta al propósito referenciado en la afirmación que caracteriza el hacer de los microempresarios.

Por otra parte, la expresión "Con frecuencia trabajo para obtener más control”, que identifica la variable poder, también posee una asociación directa con la experimentación activa de los microempresarios, es decir, que ellos aprenden haciendo cosas independientemente de cómo alcancen el poder.

\section{PRUEBA DE HIPÓTESIS}

Utilizando la prueba ji-cuadrado, que evalúa la relación entre variables se evalúa Ho1; Ho2; Ho3:

Ho1: No existe diferencia entre el comportamiento de la variable motivación y el comportamiento de la variable liderazgo de los microempresarios pertenecientes a la Incubadora de Microempresas.

El valor de ji-cuadrado calculado es de 13.27619048, por lo tanto, como el valor calculado es mayor al valor crítico se rechaza Ho1, lo cual implica que existen diferencias entre el comportamiento de la 
variable motivación y el comportamiento de la variable liderazgo.

Ho2: No existe diferencia entre el comportamiento de la variable motivación y el comportamiento de la variable aprendizaje, de los microempresarios pertenecientes a la Incubadora de Microempresas de la Universidad de Talca.

El valor de ji-cuadrado calculado es de 19.06266667, en consecuencia, como el valor calculado es mayor al valor crítico se rechaza $\mathrm{Ho2}$, lo que nos dice que existen diferencias entre los comportamientos de las variables motivación y aprendizaje.

Ho3: No existe diferencia entre el comportamiento de la variable liderazgo y el comportamiento de la variable aprendizaje de los microempresarios pertenecientes a la Incubadora de Microempresas de la Universidad de Talca.

El valor de ji-cuadrado calculado es de 15.21489621, y dado que el valor calculado es mayor al valor crítico se rechaza $\mathrm{Hoz}_{\text {, }}$ entonces se puede decir, que existe diferencia entre el comportamiento de las variables liderazgo y aprendizaje.

DISCUSIÓN

En torno al liderazgo, los microempresarios que participan en la incubadora de empresas, presentan una tendencia hacia la teoría Y (94\%), es decir, que tienen una actitud positiva hacia el trabajo y hacia sus subordinados, les agrada trabajar si las condiciones son favorables ejercen mucho autocontrol y autodirección sobre su desempeño, pueden ser muy creativos en su trabajo y en la toma de decisiones (Barbosa, et al., 2017). Además, le motivan el hacer un buen trabajo y la oportunidad de establecer relaciones amistosas con sus trabajadores, confiar en ellos más que obtener recompensas monetarias (Cabaleiro, et al. 2017).

Sin embargo, un 6\% de los encuestados presentaron una tendencia hacia la teoría $\mathrm{X}$, representativa de quienes no les gusta el trabajo, siempre que pueden procuran evitarlo y también evitan la responsabilidad (Craik, 1966), razón por la cual dele- gan responsabilidades o persuaden a sus subordinados para que realicen el trabajo (Duran y Castañeda, 2015). Generalmente, están motivados por el logro de obtener más bien y simplemente, incentivos monetarios.

Respecto del liderazgo que requieren como subordinados, la mayor tendencia se presenta en la relación baja tarea-alta relación $(62 \%)$, donde su comportamiento debería ser participativo, en el cual el líder comparte con los subordinados la toma de decisiones (Hersey y Blanchard, 1982). En esta categoría de subordinados, la tarea del líder es apoyar y motivar a sus trabajadores porque ellos presentan una madurez en la que tienen las capacidades para realizar sus tareas pero no quieren porque no están motivados (Dvir, et al., 2002).

Respecto de los estilos de liderazgo (Escalona-Barbosa y Hurtado-Ayala, 2016), se puede decir que existe una tendencia muy positiva puesto que los microempresarios ven en sus subordinados una gran capacidad para realizar sus tareas por lo que trabajan en conjunto al tomar decisiones y realizar las distintas tareas que cada uno de ellos tiene (Espinosa, et al., 2015). En consecuencia, los microempresarios deben motivar a sus trabajadores demostrándoles confianza, compartiendo con ellos una buena comunicación (Fiedler, 1987). Esta buena comunicación logra alcanzar un liderazgo participativo (Garcia, 2015), es decir, al momento de tomar decisiones en conjunto con los trabajadores se desarrolla la teoría del liderazgo continuo, donde el tipo de liderazgo se forma por la conducta del gerente, del subordinado y de la situación (Gil e Ibarra, 2014 a; 2014 b).

Con relación a los factores que motivan a los microempresarios se determinó que el término "logros" los representa como personas emprendedoras (Garcia, 2015). Tienen un fuerte deseo por encontrar soluciones a los problemas que se les presente, toman riesgos calculados y tienen una gran preocupación por lograr las metas y tareas que se han propuesto. Según esto, las personas alcanzaran sus metas siempre y cuando se sientan motivados por desarrollar actividades que le ayudan a lograrlas, como los microempresarios en su mayoría se sienten motivados por los lo- 
gros, entonces actúan positivamente en el desarrollo de sus actividades rutinarias consiguiendo así alcanzar sus metas (Godoy y Bresó, 2013).

La expectativa de los microempresarios es alta, ya que uno de los componentes era además el de trabajar duro, por lo tanto el microempresario percibe que si trabaja más duro, producirá más (Li y Liao, 2014). La valencia en ningún caso es igual a cero, porque los microempresarios perciben el valor de obtener los resultados significativos y positivos (Liao y Chuang, 2004). Además, en cuanto a la instrumentalidad el microempresario sabe que al lograr un buen desempeño podrá obtener los resultados esperados y por esto trabaja con más voluntad (Lim y Ployhart, 2004).

Respecto del aprendizaje (Kolb, 1977), el estilo que se detectó en primera mayoría en los microempresarios es el del estilo divergente que representa la gran capacidad imaginativa y la de lograr descubrir los problemas y las oportunidades (Li y Liao, 2014). Tiene también la capacidad de entablar relaciones cercanas con personas debido a que es muy sensible y se preocupa por ellas. Como punto débil se encuentra que al tener tantas ideas, le es difícil decidirse por alguna (Lim y Ployhart, 2004).

El segundo estilo que se encontró fuertemente entre los microempresarios fue el acomodador, que tiene como punto fuerte el ser líder innato, puede lograr resolver problemas y establecer buenas relaciones, siempre y cuando estas relaciones le ayuden a lograr sus objetivos, es decir que sabe influir en las personas para que hagan lo que ellos quieren (Morelos-Gomez y Fontalvó-Herrera, 2014).

En el aprendizaje social de los microempresarios predomina el medio, debido a que las relaciones con sus trabajadores son altas, logrando entrelazar los principales componentes para alcanzar un aprendizaje basado en la experiencia directa que ayudara en la conducta futura del microempresario (Moreno, et al., 2014).

Acerca de las hipótesis, se puede anali- zar que existen diferencias entre las variables que se sometieron al estudio entre los microempresarios pertenecientes a la Incubadora de Microempresas, lo que nos indica que las tres variables Liderazgo, Motivación y Aprendizaje, y sus componentes, son individualmente significativas y relevantes para caracterizar los modelos mentales de los microempresarios, es decir, las variables tienen una distinta explicación para cada microempresario (Pautt, 2011). Sin embargo, aún, existiendo muchas y diversas similitudes entre sus comportamientos, cada microempresario tiene una distinta interpretación para determinar qué componentes de cada variable es la más relevante para cada quién (Pedroja-Rejas y Rodriguez-Ponce, 2008).

\section{CONCLUSIONES}

Respecto del aprendizaje, si bien existen similitudes entre los microempresarios, no todos presentan el mismo estilo, además, los métodos que definen los estilos, no presentan la misma importancia. Se verifica que el estilo más recurrente es el divergente seguido del acomodador y del asimilador, terminando con el convergente.

En general, los microempresarios, se motivan por los logros, pero aun así los componentes que integran a esta variable son interpretados de distinta forma por los microempresarios y contribuye a la auto - comprensión que los microempresarios alcanzan de sus propias conductas, por cuanto, existen similitudes en los estilos de liderazgo y la mayor motivación se relaciona con el logro.

Considerando que los micro empresarios buscan con frecuencia optimizar sus actividades, las opciones que tienen para lograrlo implican identificar cuáles serían las acciones que, de manera concatenada, debieran realizar, primero, reconociendo el aprendizaje que en términos concretos y prácticos ellos alcanzan, segundo, asociar estos aprendizajes con los conceptos teóricos que dominan y, finalmente, estar conscientes de cuáles son las actitudes y conductas más eficaces para la toma de decisiones. 


\section{REFERENCIAS}

Barnard, C.I. (1938) The Functions of the Executive. Cambridge, MA : Harvard University Press.

Hersey, P. \& Blanchard, K.H. (1982). Leadership style: attitudes and behavior. Training and Development Journal, 36 (2), 50-52.

Avolio, B. J. y Bass. B. M. (2004) Multifactor leadership questionnaire: Manual and sample set (3rd ed.) Redwood City, ca: Mind Garden

Bandura, Albert. (1982) Aprendizaje Social. Ed. Espasa Calpe. Madrid. España. Disponible en: www.galeon.com/pcazau/ resps_bandu.htm

Barbosa R. D. H., Mihi R. A., Noguera H. Á. (2014) Knowledge management and leadership: Relationship prospects. Perspect. Psicol. ISSN: 1794-9998 (en línea), Vol. 10, No. 1, pp. 057-070

Cabaleiro G., Jiménez, A., Miles, J. y Horta R. (2016) Liderazgo e innovación para un mundo más sostenible. Journal of Technology Managemente \& Innovatión ISSN 0718-2724 vol.11 no.1 Santiago

Craik, Kenneth J. W. (1966) Sherwood, Stephen L., ed. The Nature of Psychology: A Selection of Papers, Essays and Other Writings by Kenneth J. W. Craik. Cambridge: Cambridge University Press. ISBN 978-0521134804.

Duran, M. G. y Castañeda D. I. (2015) Relación entre Liderazgo Transformacional y Transaccional con la Conducta de compartir conocimiento en dos empresas de servicios. Acta Colombiana de Psicología 18.1

Dvir, T., Eden D., Avolio B. J. y Shamir. B. (2002) Impact of transformational leader ship on follower development and performance: A field experiment. doi. org/10.2307/3069307. Academy of Management Journal, 45(4), 735-744

Ehrhart, M. G., (2004) Leadership and procedural justice climate as antecedents of unit-level organizational citizenship behavior. doi.org/10.1111/j.1744-6570.2004. tbo2484.x. Personnel Psychology, 57, 6194

Escalona-Barbosa, D., Hurtado-Ayala, A. (2016) Influencia de los estilos de liderazgo en el desempeño de las empresas exportadoras de colombia. Estudis Genera- les 32.139

Espinosa, J., Contreras F. y Barbosa D. (2015) Prácticas de Liderazgo y su relación con la Cultura en un grupo de paises Latinoamericano. 11.2

Eyssautier M. (2006) Metodología de la investigación. México D.F.: Editorial Thomson Fiedler, Fred. (1987) A Theory of Leadership Effectiveness. New York. U.S.A. Ed. Mc Graw Hill. Disponible en: www.teclaedo.edu.mx/unidad4/modelode.htm

Garcia, S. M. (2015) Papel de los Seguidores en el desarrollo de las teorías de liderazgo Organizacional. Apuntes del Cenes 34.59

Gil, O. I. e Ibarra, L. S. (2014 a) Impact Of Leadership In Critical Success Factors As A Competitive Business Strategy. Dimensión Empresarial / Vol. 12 No. 2 / Julio - Diciembre, págs. 117-126

Gil, O., I. e Ibarra, L. S. (2014 b) Incidencia del liderazgo en los factores críticos del éxito como estrategia competitiva empresarial. Dimension Empresarial 12.2.

Codoy, R, y Bresó, E. (2013) ¿Es el liderazgo transformacional determinante en la motivación intrinseca de los seguidores? Revista de psicología del trabajo y de las organizaciones 29.2

Hernández, R., Fernández, C. y Baptista, P. (2006) Metodología de la Investigación. México D. F.: Mc Graw-Hill

IBM (2013) Statistical Package for the Social Sciences, SPSS Statistics Base 20V

Kolb, David. (1977). "Psicología de las Organizaciones”. Editorial Prentice-Hall Hispanoamericana, S.A. México.

Li, A. N. y Liao, H., (2014) How do leader-member exchange quality and differentiation affect performance in teams? An integrated multilevel dual-process model. Journal of Applied Psychology, 99, 847-866

Liao, H. y Chuang, A., (2004) A multilevel investigation of factors influencing employee service performance and customer outcomes. Academy of Management Journal, 47, 41-58

Lim, B. y Ployhart, R. (2004) Transformational leadership: Relations to the five-factor model and team performance in typical and maximum contexts. Journal of Applied Psychology, 89, 610-621

Morelos-Comez, J. y Fontalvó-Herrera T., (2014) Análisis de los factores determinantes de la Cultura Organizacional en el Ambiente Empresarial, 10.1

Moreno, M., Navarro, C. y Humanes, M., 
(2014) El liderazgo en relaciones públicas y gestión de comunicación. Análisis cuantitativo de los factores de liderazgo en el sector en España. Palabra Clave, vol. 17 no. 3 Chia Sept. / Dec.

Pautt T. G. (2011) Liderazgo y Dirección: Dos Conceptos Distintos Con Resultados Diferentes. Rev. Fac. Cienc. Econ., ISSN: 01216805 Vol. XIX (1), Junio, 213-228

Pedroja-Rejas, L. y Rodriguez-Ponce E., (2008) Estudio comparativo de la influencia del estilo de liderazgo y la congruencia de valores en la eficacia de empresas privadas e instituciones públicas. Interciencia 33.1

Perez L. S., (2012) La influencia del liderzgo sobre el aprendizaje. El papel mediador del contexto organizativo. Innovar, 22.45

Pérez S. J. y Camps V., (2011) Transformatio- nal Leadership Manifestation in a Group of Puertorrican Supervisors. Rev. psicol. trab. organ. vol.27 no.1 Madrid abr.

Robbins, Stephen (1993) Comportamiento Organizacional Sexta edición Ed. Mc Graw Hill, México.

Robbins, Stephen P. (1996) Comportamiento Organizacional. Teoría y Práctica. Prentice Hall, 7 ma., edición

Senge, Peter, (1990) La Quinta Disciplina. Ed. Vergara/Granica. Buenos Aires. Argentina.

Zárate T. R. y Matviuk S., (2012) Emotional intelligence and leadership practices in Colombian Organizations. Cuadernos de Administración, Universidad del Valle, Volumen 28 No. 47 enero - junio. 